VII JORNADA DE DIFUSIÓN DE LA INVESTIGACIÓN Y EXTENSIÓN - FCV-UNL

RESUMEN EXTENDIDO

\title{
ESTIMACIÓN DE UNA DOSIS DE MARBOFLOXACINA PARA CABRAS DE TRES SEMANAS DE EDAD MEDIANTE ANÁLISIS POBLACIONAL.
}

\author{
Dell'Elce $A^{1}$, Anadón $A^{1}$, Menseguéz $S^{1}$, Castromán $R^{1}$, Ferrer $A^{1}$, Rubio $G^{2}$, Bertero $\mathrm{J}^{2}$, Candioti $\mathrm{V}^{2}$, \\ Fernández $\mathrm{H}^{1}$, Picco $\mathrm{E}^{1}$, Botto $\mathrm{M}^{3}$, Formentini $\mathrm{E}^{1}$.
}

${ }^{1}$ Laboratorio de Farmacología y Toxicología, FCV-UNL

${ }^{2}$ Cátedra de Semiología, FCV-UNL

${ }^{3}$ Cátedra de Bioestadística, FCV-UNL

* Correspondencia: Dell'Elce A., E-mail: adellelce95@gmail.com

Editado por: R. Sobrero, V. Matiller, C. Baravalle

\section{ESTIMATION OF A DOSE OF MARBOFLOXACIN FOR 3-WEEK-OLD GOATS BY POPULATION ANALYSIS.}

SUMMARY.

A dose of marbofloxacin (MFX) to treat gastrointestinal infections caused by E. coli in 3-week-old goats was estimated. The pharmacodynamics of MFX against E. coli was evaluated in vitro by estimation of minimum inhibitory concentration (MIC), minimum bactericide concentration (MBC) and mutant prevention concentration (MPC). Marbofloxacin was administered to six 3-week-old goats by subcutaneous route at the dose of $2 \mathrm{mg} / \mathrm{kg}$. The pharmacokinetic parameters were estimated by non-compartmental analysis. The dose of MFX capable to protect the $95 \%$ of population was calculated considering the population distribution of pharmacokinetic parameters. The efficacy of MFX was evaluated by the relationship between the area under curve and MPC (AUC/MPC) with a cut-off value of $22 \mathrm{~h}$. The results showed that the estimated dose of MFX to reach the clinical outcome of gastrointestinal infections caused by E. coli and to prevent the bacterial resistance at the $95 \%$ of the population of 3-week-old goats was $3,179 \mathrm{mg} / \mathrm{kg}$, which for practical reasons was fixed at $3,5 \mathrm{mg} / \mathrm{kg}$. The in vivo efficacy of dose estimated will be tested in future studies.

Palabras clave: Marbofloxacina, Escherichia coli, Cabras, Dosis

Keywords: Marbofloxacin, Escherichia coli, Goats, Dose

La marbofloxacina (MFX) es una fluoroquinolona (FQ) desarrollada para ser usada en medicina veterinaria. Presenta actividad sobre cepas de Pasteurella multocida, Mannheimia haemolytica, Mycoplasma bovis y Escherichia coli (E. coli). Se ha propuesto el uso de MFX para el tratamiento de infecciones gastrointestinales en terneros causadas por E. coli. No se dispone de dosis de MFX para especies productivas menores como la caprina, por lo que el uso en esta especie se realiza extra rótulo utilizando el esquema posológico extrapolado del bovino ( $2 \mathrm{mg} / \mathrm{kg} \mathrm{c} / 24 \mathrm{~h}$ durante 3-5 días consecutivos). Se acepta que una terapéutica antibiótica óptima debe garantizar la remisión clínica, la erradicación bacteriana y prevenir la emergencia de resistencia. Esto último ha hecho que para las (FQs) entre en vigencia un nuevo parámetro farmacodinámico (PD): la concentración preventiva de mutantes (CPM). Sin embargo las dosis actuales de las FQs han sido estimadas considerando la concentración inhibitoria mínima (CIM) y no a la CPM. EI objetivo de este trabajo fue realizar un estudio farmacocinético (PK) de MFX en cabras de tres semanas de edad y a partir de un análisis farmacocinéticofarmacodinámico (PK-PD) poblacional estimar la dosis de MFX para tratar infecciones causadas por $E$. coli para 
garantizar la eliminación del agente infeccioso y prevenir la emergencia de cepas resistentes en el $90 \%$ de una población tratada. Se utilizó una cepa estandarizada de E. coli (ATCC 25922), un estándar de MFX de pureza conocida y una solución inyectable experimental de MFX al $10 \% \mathrm{p} / \mathrm{v}$. Se determinaron los valores de CIM y de CPM (Wetzstein, 2005). El estudio de PK de MFX en cabras de tres semanas de edad se realizó en la Unidad Académico Experimental (UAE) de la FCV-UNL con aprobación del Comité de Ética y Seguridad (CAES) de la FCV-UNL. Una dosis de MFX de $2 \mathrm{mg} / \mathrm{kg}$ se administró por vía SC a 6 cabras de raza Boer de tres emanas de edad. Se extrajeron muestras de sangre a tiempos preestablecidos y la cuantificación de MFX en plasma se realizó mediante método microbiológico. El análisis PK se realizó mediante análisis no compartimental. A partir de los valores promedio y desvíos estándar de cada parámetro PK se calcularon los valores de los límites superior (LS) e inferior (LI) de los intervalos de tolerancia al 90\% (IT90\%) estimados con un intervalo de confianza al 90\% (IC90\%). Mediante simulación Monte Carlo se realizaron las distintas combinaciones posibles de los $\mathrm{LI}$ y LS de los parámetros PK, realizándose la simulación de todos los perfiles posibles de concentración plasmática, identificándose las combinaciones de los parámetros PK que originaron el mayor y el menor perfil de concentración plasmática. Las simulaciones se realizaron con el software ADAPT II (BMRS, University of Southern California, USA) y de cada perfil simulado se estimaron los correspondientes parámetros PK. A partir de los valores de los parámetros PK que originaron el menor perfil de concentración plasmática se calculó con la ecuación 1, la dosis de MFX capaz de lograr una relación $A B C 24 / C P M \geq 22 h$, que es la que se considera adecuada para obtener la resolución del cuadro clínico y prevenir la emergencia de cepas de $E$. coli resistentes a MFX.

$$
\text { Dosis }=\frac{C P M \cdot 22 \cdot(C L / F)}{f_{u}}
$$

Donde CPM es la concentración preventiva de mutantes de la cepa bacteriana en estudio, 22 es el valor de la relación $A B C 24 / C P M$ para prevenir la emergencia de resistencia, fu es la fracción no unida a proteínas plasmáticas y $\mathrm{CL} / \mathrm{F}$ es el valor del aclaramiento corporal (CL) estimado por vía extravascular corregido por la fracción biodisponible $(\mathrm{F})$ estimado como ke $\times(\mathrm{Vd} / \mathrm{F})$; donde ke es la constante de eliminación de primer orden aparente, $\mathrm{Vd} / \mathrm{F}$ es el volumen de distribución estimado por vía extravascular corregido por la fracción biodisponible (F). Los valores de CIM y de CPM de E. coli ATCC 25922 fueron de 0,0312 y $0,1 \mu \mathrm{g} / \mathrm{mL}$ respectivamente. Sin embargo para el cálculo de la dosis de MFX se utilizó como factor de seguridad el valor de CPM90 de $0,5 \mu \mathrm{g} / \mathrm{mL}$ reportado en la literatura (CLSI, 2008). El perfil de concentración plasmática de MFX en cabras de tres semanas de edad se presenta en la figura 1.
Los parámetros PK de MFX estimados a partir del análisis no compartimental de los parámetros PK promedio y los correspondientes índices PK-PD resultantes se presentan en la tabla 1.

La dosis de $2 \mathrm{mg} / \mathrm{kg}$ solamente protege al perfil promedio de la población (Tabla 1 ). Por esa razón se estimó una dosis de MFX de $3077,0 \mu \mathrm{g} / \mathrm{kg}(3,077 \mathrm{mg} / \mathrm{kg})$ que permitió lograr una relación $A B C 24 / C P M \geq 22$ h para todas las combinaciones posibles de los valores de LI y LS de los IT90\% de los parámetros PK de MFX. Los perfiles plasmáticos simulados de MFX (menor y mayor) obtenidos con la dosis estimada de $3,077 \mathrm{mg} / \mathrm{kg}$ se presentan en la figura 2 .

Según los resultados obtenidos, una dosis de MFX de $3077,0 \mu \mathrm{g} / \mathrm{kg}$ sería suficiente para lograr sobre el $90 \%$ de una población de cabras de tres semanas de vida valores de $A B C 24 / C P M \geq 22,0 \mathrm{~h}$ necesarios para garantizar la remisión clínica de cuadros infecciosos provocados por E. coli y prevenir la emergencia de cepas resistentes. Sin embargo, a los fines de facilitar la dosificación proponemos utilizar una dosis de $3000 \mu \mathrm{g} / \mathrm{kg}(3 \mathrm{mg} / \mathrm{kg})$. Los resultados obtenidos en este estudio serán utilizados para la realización de ensayos de mayor magnitud para testear la eficacia de la dosis propuesta.

Figura 1. Perfil de concentración plasmática de MFX tras su administración SC a cabras de tres semanas de edad a la dosis de $2 \mathrm{mg} / \mathrm{kg}$. Los valores se hallan expresados como promedio y desvío estándar.

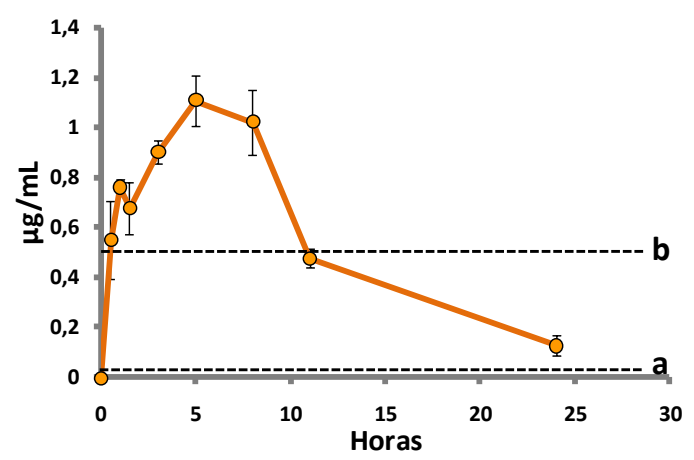




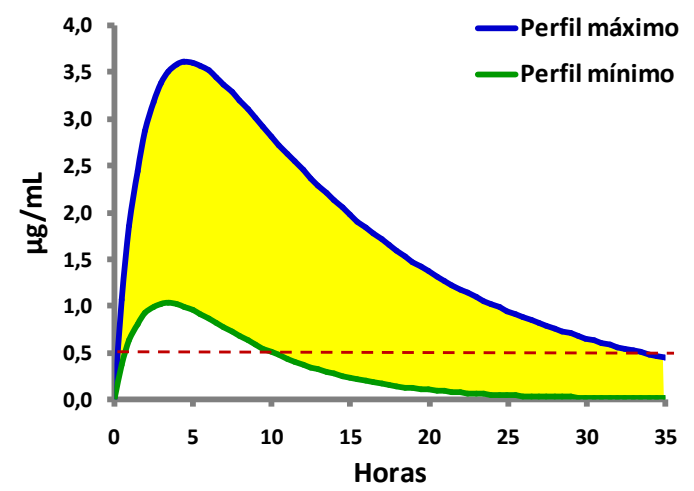

Figura 2. Gráfica del área de probabilidad de ocurrencia de un perfil de concentración plasmática de MFX en el $90 \%$ de una población de cabras de tres semanas de vida tras la administración SC de una dosis de $3077,0 \mu \mathrm{g} / \mathrm{kg}$. El área de probabilidad sombreada en amarillo se halla delimitada por un perfil de concentración plasmática máximo que se obtuvo con los valores de $\mathrm{LI}$ de $\mathrm{Vd} / \mathrm{F}$ y ke, mientras que el perfil mínimo se obtuvo con los valores de LS de Vd/F y ke. La línea de puntos horizontal a indica la CPM90 $(0,5 \mu \mathrm{g} / \mathrm{mL})$.

Tabla 1. Parámetros PK y PK-PD de MFX tras su administración subcutánea (SC) a la dosis de $2 \mathrm{mg} / \mathrm{kg}$ en cabras de tres semanas de vida. Donde ka es la constante de absorción, tmax es el tiempo de la máxima concentración plasmática ( $\mathrm{Cmax}$ ) y $\mathrm{ABC} \infty$ es el área bajo la curva de concentración plasmática extrapolada al infinito. El resto de los símbolos fueron explicados anteriormente. $\left({ }^{*}\right)$ Con un intervalo de administración cada $24 \mathrm{~h}$ el valor del $\mathrm{ABC} \infty$ es equivalente al ABC24

\begin{tabular}{lcc}
\hline Parámetros & Promedio & $\mathrm{DE}$ \\
\hline $\mathrm{k}_{\mathrm{a}}\left(\mathrm{h}^{-1}\right)$ & 0,41 & 0,031 \\
$\mathrm{k}_{\mathrm{e}}\left(\mathrm{h}^{-1}\right)$ & 0,122 & 0,020 \\
$\mathrm{t}_{\max }(\mathrm{h})$ & 6 & 1,54 \\
$\mathrm{C}_{\max }(\mu \mathrm{g} / \mathrm{mL})$ & 1,14 & 0,12 \\
$\mathrm{~V}_{\mathrm{d}} / \mathrm{F}(\mu \mathrm{g} / \mathrm{mL})$ & 1165,7 & 236,5 \\
$\mathrm{ABC} \mathrm{C}_{\infty}(\mu \mathrm{g} \cdot \mathrm{h} / \mathrm{mL})$ & 14,5 & 1,44 \\
$\mathrm{ABC}_{\infty} / \mathrm{CPM}^{*}(\mathrm{~h})$ & 29,1 & 2,95 \\
\hline
\end{tabular}

\section{Bibliografía}

CLSI (Clinical and Laboratory Standards Institute). 2008. Development of in vitro susceptibility testing criteria and quality control parameters for veterinary antimicrobial agents; Approved Guideline. 3rd Edition, Document M37-A3, Volume 28, Number 7, Wayne, Pennsylvania USA.

Wetzstein HG. 2005. Comparative mutant prevention concentrations of pradofloxacin and other veterinary fluoroquinolones indicate differing potentials in preventing selection of resistance. Antimicrob. Agents. Ch. 49(10): 41664173. 